\title{
Mechanical behavior and thermal activation analysis of HfNbTaTiZr body-centered cubic high-entropy alloy during tensile deformation at
} $77 \mathrm{~K}$

\author{
Rajeshwar R. Eleti*, Nikita Stepanov, Sergey Zherebtsov \\ Laboratory of Bulk Nanostructured Materials, Belgorod National Research University, Pobeda 85, Belgorod 308015, Russia
}

\section{A R T I C L E I N F O}

\section{Article history:}

Received 6 June 2020

Revised 5 July 2020

Accepted 11 July 2020

Available online 22 July 2020

\section{Keywords:}

Low-temperature plasticity

Body-centered cubic

High-entropy alloy

Slip trace analysis

Activation volume

\begin{abstract}
A B S T R A C T
Plastic deformation of HfNbTaTiZr body-centered cubic high-entropy alloy (BCC-HEA) was investigated at $77 \mathrm{~K}$. Tensile stress-strain curves showed strong temperature dependence of yield strength. Characteristics of dislocation slip at $77 \mathrm{~K}$ was analyzed using slip traces which confirmed active slip on $\{112\}$ planes. The apparent activation volume $\left(V^{*}\right)$ suggested thermally activated kink/kink-pair nucleation at $77 \mathrm{~K}\left(V^{*} \sim 7 \mathrm{~b}^{3}\right)$. $V^{*}$ values of BCC-HEA and pure Nb were nearly same at $77 \mathrm{~K}$. Low-temperature plasticity of the BCC-HEA showed characteristics of pure BCC metals, suggesting chemical-complexity of HEAs may not alter the Peierls potential of $a / 2<111>$ screw dislocations.
\end{abstract}

(C) 2020 Acta Materialia Inc. Published by Elsevier Ltd. All rights reserved.
Body-centered cubic (BCC) lattice is a loosely packed crystal structure unlike face-centered cubic (FCC) or hexagonal-close packed (HCP) structures. Due to this, BCC crystals lack close packed planes. However, $\{110\}$ planes of BCC crystals are considered as the closest packed planes having $<111>$ close packed directions. Further, $\{112\}$ and $\{123\}$ planes equally contain the $<111>$ slip direction. Consequently, the core of screw dislocations may spread onto multiple crystallographic planes along the $<111>$ direction. Thus, plastic deformation of BCC metals and alloys is primarily controlled by the motion of $a / 2<111>$ screw dislocations [1-3]. As a result of this non-planar dislocation core structure, low-temperature deformation of BCC crystals is characterized by a remarkable increase in the strength $[4,5]$. Adding to that, the long, straight screw dislocations will not be able to move independently through the lattice which may facilitate the thermally activated kink/kink-pair nucleation [1]. As kinks being only few atomic widths, however, the motion of kinks yields low apparent activation volume $\left(V^{*}\right)$. Since $V^{*}$ is directly related to dislocation movement, its evaluation can provide insights into the rate-controlling dislocation mechanism [6].

Caillard and Martin [6], Seegar and Holzwarth [7], Conrad [8], Werner [9], Basinski and Christian [10], Hoge and Mukherjee [11] had previously studied, and reviewed the temperature dependence of strength and also reported the thermally activated pro-

\footnotetext{
* Corresponding author.

E-mail address: rajeshwar.eleti@gmail.com (R.R. Eleti).
}

cesses of BCC crystals. It has been suggested that most of the BCC metals and alloys behave rather similar. That is, the plastic flow due to screw dislocation motion is controlled by overcoming the Peierls-Nabarro stress barrier at $300 \mathrm{~K}$ and the kink/kink-pair nucleation at $77 \mathrm{~K}$. Recently, in complex alloys like high-entropy alloys (HEAs) [12], screw dislocation strengthening has been energetically considered [13-15]. Maresca and Curtin [15] proposed the long, straight screw dislocations of BCC-HEAs would naturally form a kink-structure to avoid the high-energy hills. However, systematic analysis on the low-temperature plasticity of BCC-HEAs is still missing, unlike the FCC-HEAs [16,17].

The equiatomic HfNbTaTiZr is the most studied BCC-HEA [18-27]. Juan et al. [28] reported that the strength (>900 MPa) and ductility ( $>10 \%$ ) of HfNbTaTiZr increased with decrease of grain size, during tensile deformation at room temperature. Chen et al. [29] studied the dependence of the yield strength and flow behavior of this alloy during tensile deformation at ambient temperatures. They reported the yield strength increased with decrease of temperature. Although mechanical properties of HfNbTaTiZr are attractive, dislocation slip behavior of HfNbTaTiZr BCC-HEA has not been well understood yet. Further investigations are required to understand the intricacies of the plastic flow to realize the full potential of this alloy. Moreover, mechanical behavior of BCC-HEAs at cryogenic temperatures is unknown so far. The main objective of the present study is therefore, to systematically study the characteristics of dislocation slip and investigate thermal activated analysis of HfNbTaTiZr BCC-HEA at liquid nitrogen $(77 \mathrm{~K})$ temperature. 
The vacuum arc-melted, as-cast HfNbTaTiZr alloy was coldrolled until $86 \%$ reduction and annealed at $1200^{\circ} \mathrm{C}$ for 5 to $20 \mathrm{~min}$, and subsequently water quenched. Tensile specimens having gauge dimensions $6 \times 3 \times 1.2 \mathrm{~mm}^{3}$ were cut from the annealed sheets. The rolling direction of the cold-rolled sheet was aligned parallel to the longitudinal axes of the tensile specimens. Tensile tests were carried out at $300 \mathrm{~K}$ and $77 \mathrm{~K}$ for the constant strain-rate $10^{-3} s^{-1}$. Interrupted tensile tests were performed for clarifying the microstructure evolution and investigating slip trace analysis. Further, stress relaxation tests during tensile deformation were performed to investigate dislocations slip related activation parameters. Microstructural observations were performed using electron back-scattering diffraction (EBSD) and back-scattering electron (BSE) imaging systems operated in a field emission-scanning electron microscope (FE-SEM, FEI-Nova NanoSEM 450).

Annealing the cold-rolled sheet at $1200{ }^{\circ} \mathrm{C}$ for different time intervals has produced fully recrystallized microstructures having various grain sizes, $d=10-60 \mu \mathrm{m}$ (refer supplementary materials). Fig. 1(a) shows of the microstructure of HfNbTaTiZr alloy having average grain size, $d \sim 60 \mu \mathrm{m}$. Fig. 1(b) shows the engineering stress-strain curves of the HfNbTaTiZr alloy during tensile deformation at $300 \mathrm{~K}$ and $77 \mathrm{~K}$. Firstly, decrease of the deformation temperature increased the yield strength $\left(\sigma_{0.2 \%}\right)$ from $984 \mathrm{MPa}$ at $300 \mathrm{~K}$ to $1638 \mathrm{MPa}$ at $77 \mathrm{~K}$, for $d \sim 60 \mu \mathrm{m}$. Second, decrease of grain size increased the $\sigma_{0.2 \%}$ but decreased ductility at $77 \mathrm{~K}$, contrary to $300 \mathrm{~K}[23,28]$. The increase in $\sigma_{0.2 \%}$ is due to the wellknown Hall-Petch effect [30];

$\sigma_{0.2 \%}=\sigma_{0}+k_{H P} d^{-0.5}$

where $\sigma_{0}$ is friction stress (y-intercept), $k_{\mathrm{HP}}$ is Hall-Petch coefficient (slope), $d$ is average grain size. The Hall-Petch relationship for yield strength and grain size at $77 \mathrm{~K}$ was plotted and compared with the room temperature data of Juan et al. [28] (Fig. 1(c)). It was clear that the decrease of temperature increased $\sigma_{0}$ and $k_{\mathrm{HP}}$ values. Here, it is noteworthy that temperature dependence of the $\sigma_{0.2 \%}$ of the BCC-HEA is consistent with the non-planar dislocation cores of BCC crystals. This aspect is due to decrease of thermal fluctuations mandates an increase of stress assistance for the movement of dislocation across the Peierls-Nabarro stress barrier (friction stress). At low-temperatures, the increase in $\sigma_{0.2 \%}$ naturally increases the Hal-Petch intercept. That is, the increased $\sigma_{0}$ (y-intercept) is merely due to increased $\sigma_{0.2 \%}$ associated with the non-planar dislocation core structure. On the other hand, $k_{\mathrm{HP}}$ value showed nearly three-fold increment from $240 \mathrm{MPa} \mu \mathrm{m}^{0.5}$ at $300 \mathrm{~K}$ to $656 \mathrm{MPa} \mu \mathrm{m}^{0.5}$ at $77 \mathrm{~K}$, which indicated an increase of grain boundary (GB) strengthening. This aspect is discussed in the later paragraphs.

Interrupted tensile test was carried out on pre-polished specimen having $d \sim 60 \mu \mathrm{m}$, in order to investigate and clarify the characteristic deformation mechanisms at $77 \mathrm{~K}$. Fig. 2(a-c) shows the EBSD-IPF map and SEM-BSE images of HfNbTaTiZr deformed at $77 \mathrm{~K}$, until strain, $e \sim 2.2 \%$, observed along the longitudinal direction. The BSE images revealed straight slip traces. Fig. 2(d-f) shows the EBSD-IPF map and SEM-BSE images of the same sample observed along the lateral direction. We further estimated the Schmid factor $(m)$ of those slip traces and showed along the corresponding reference white lines of Fig. 2(c and f). For comparison, we identified two grains, G1 and G2 having nearly the same orientation, closer to [001] of the standard stereographic triangle. The characteristics of straight slip traces and numerical $m$ values of $G 1$ and G2 perfectly agreed with each other. Using the EBSD data, we further evaluated $m$ values for the conventional slip systems of BCC crystals. Here, we showed a case study for G1 and G2 having orientations [105] and $[5,1,25] / /$ T.A., respectively. The $m$ values determined for various slip systems are shown in Table 1 . For G1 and G2, the slip system (-112)[1-11] showed the highest $m$ value. (a)
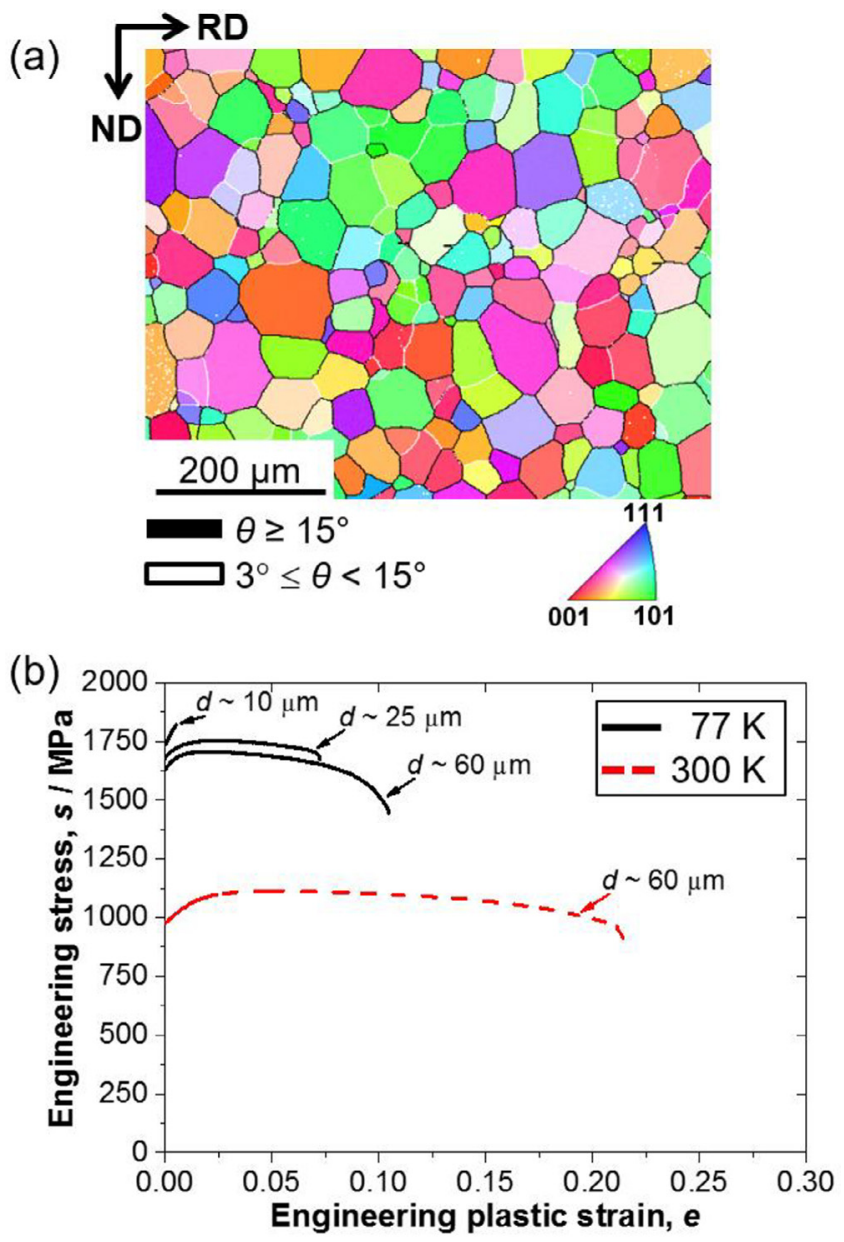

(c)

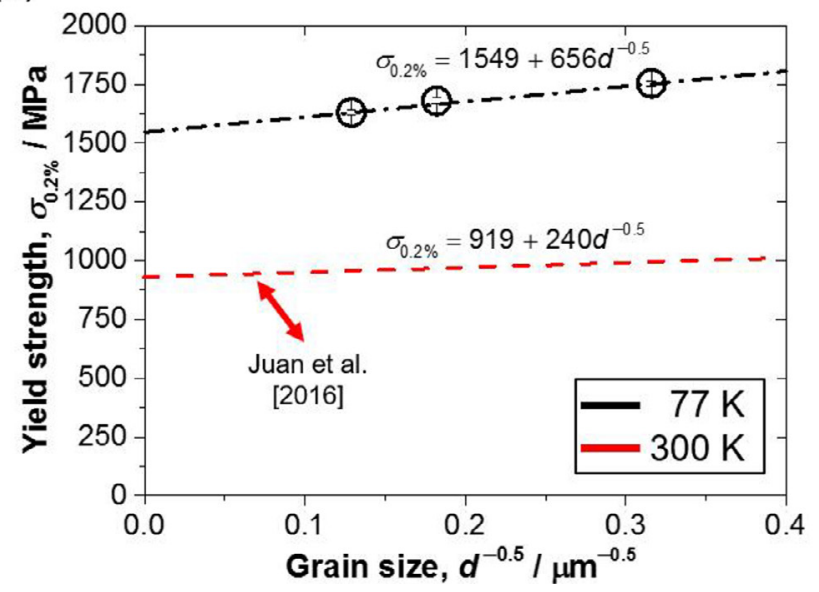

Fig. 1. Microstructure and stress-strain curves of HfNbTaTiZr during tensile deformation at $300 \mathrm{~K}$ and $77 \mathrm{~K}$ for the strain rate $10^{-3} \mathrm{~s}^{-1}$. (a) EBSD-IPF map of fully recrystallized microstructure having grain size, $d \sim 60 \mu \mathrm{m}$. (b) Tensile stress-strain curves of HfNbTaTiZr deformed at $300 \mathrm{~K}$ and $77 \mathrm{~K}$. (c) Hall-Petch plot of HfNbTaTiZr alloy for different tensile deformation temperatures.

Although the $m$ values of slip trace analysis (Fig. 2(c and f)) perfectly coincided with the determined $m$ values of Table 1 , accurate identification of slip systems is an intricate process. To verify the exact slip system, we extended the analysis using stereographic projections. Fig. 3(a) shows SEM-BSE image of the BCC-HEA deformed at $77 \mathrm{~K}$. As was mentioned, G1 had the orientation [105]//T.A.. So, stereographic projection was plotted for the [105]//T.A. and shown in Fig. 3(b). The great circle containing slip trace along the $\langle 111\rangle$ direction was shown in the figure. As per the 
Table 1

Summary of slip systems and the corresponding Schmid factor, $m$ values for grains G1 and G2 having nearly similar orientations [ $\left[\begin{array}{lll}1 & 0 & 5\end{array}\right]$ and [ $\left.5 \begin{array}{lll}5 & 1 & 25\end{array}\right]$ respectively. Here, $m$ value was determined as the product of dot product of the applied stress direction and slip plane, and the dot product of the applied stress direction and slip direction.

\begin{tabular}{|c|c|c|c|c|c|}
\hline Orientation & Slip system & Schmid factor, $m$ & Orientation & Slip system & Schmid factor, $m$ \\
\hline G1, [ [ [ $\left.\begin{array}{lll}1 & 0 & 5\end{array}\right] / /$ T.A. & $\begin{array}{l}(011)[11-1] \\
(101)[11-1] \\
(1-10)[11-1] \\
(01-1)[1-1-1] \\
(101)[1-1-1] \\
(110)[1-1-1] \\
(011)[1-11] \\
(10-1)[1-11] \\
(110)[1-11] \\
(01-1)[111] \\
(10-1)[111] \\
(1-10)[111] \\
(2-11)[11-1] \\
(-121)[11-1] \\
(112)[11-1] \\
(211)[1-1-1] \\
(12-1)[1-1-1] \\
(1-12)[1-1-1] \\
(21-1)[1-11] \\
(121)[1-11] \\
(-112)[1-11] \\
(-211)[111]\end{array}$ & $\begin{array}{l}-0.31 \\
-0.37 \\
-0.06 \\
0.31 \\
-0.37 \\
-0.06 \\
0.47 \\
-0.37 \\
0.09 \\
-0.47 \\
-0.37 \\
0.09 \\
-0.25 \\
-0.14 \\
-0.39 \\
-0.25 \\
0.14 \\
-0.39 \\
-0.16 \\
0.33 \\
0.49 \\
0.16\end{array}$ & G2, [llllll//T.A. & $\begin{array}{l}(011)[11-1] \\
(101)[11-1] \\
(1-10)[11-1] \\
(01-1)[1-1-1] \\
(101)[1-1-1] \\
(110)[1-1-1] \\
(011)[1-11] \\
(10-1)[1-11] \\
(110)[1-11] \\
(01-1)[111] \\
(10-1)[111] \\
(1-10)[111] \\
(2-11)[11-1] \\
(-121)[11-1] \\
(112)[11-1] \\
(211)[1-1-1] \\
(12-1)[1-1-1] \\
(1-12)[1-1-1] \\
(21-1)[1-11] \\
(121)[1-11] \\
(-112)[1-11] \\
(-211)[111]\end{array}$ & $\begin{array}{l}-0.3 \\
-0.36 \\
-0.04 \\
0.31 \\
-0.39 \\
-0.07 \\
0.47 \\
-0.36 \\
0.1 \\
-0.46 \\
-0.38 \\
0.07 \\
-0.23 \\
-0.15 \\
-0.38 \\
-0.27 \\
0.13 \\
-0.41 \\
-0.14 \\
0.33 \\
0.483 \\
0.19\end{array}$ \\
\hline
\end{tabular}
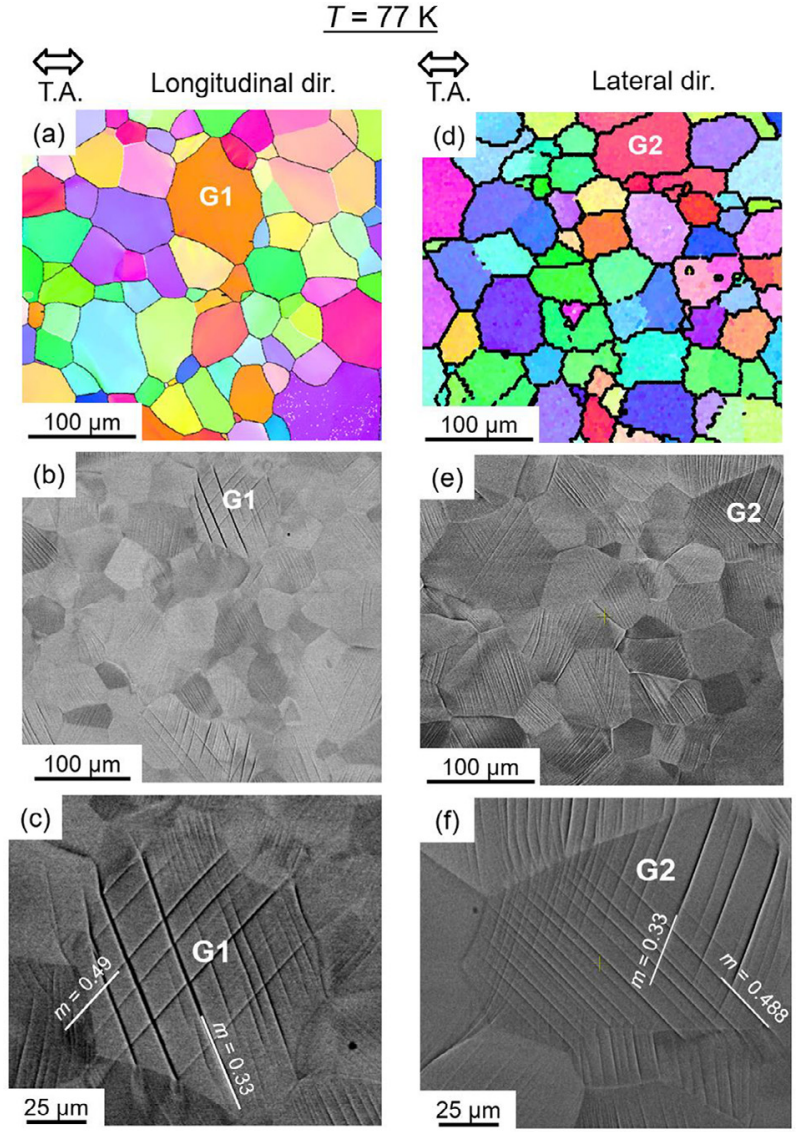

Fig. 2. Microstructures of HfNbTaTiZr after a tensile strain, $e \sim 2.2 \%$ deformed at 77 K. (a) EBSD-IPF map along the longitudinal direction (observed on gauge surface), and its corresponding BSE image shown in (b). (c) High-magnification image of a grain G1. (d) EBSD-IPF map along the lateral direction (observed on thickness surface), and its corresponding BSE image shown in (e). (f) High-magnification image of a grain G2. The Schmid factor, $m$ values of the corresponding slip traces are shown in white color. Here, the $m$ value was determined as the product of cosines of the direction along the slip trace and slip trace normal with respect to the tensile axis (T.A.). T.A. is horizontal to the microstructures. projection, the great circle matched perfectly with the experimentally observed slip trace, T1 of Fig. 3(a) along the [1-11] direction. Later, another great circle containing the slip trace normal was shown on the stereographic projection which indicated $(-112)$ and its symmetric plane (1-1-2) as the potentially active slip planes. According to the fundamentals of plasticity, dot product of the slip plane and the slip trace direction must be equal to zero for the slip trace along a direction to contain the slip plane. The obtained slip plane $(-112)$ perfectly met the criteria along the [1-11] direction. The identified slip system (-112)[1-11] was compared with the slip systems for the [105] orientation of G1 (Table 1). Perfect agreement was found for the $(-112)[1-11]$ slip system having the highest $m \sim 0.49$. Here, the notable conclusion is that the dislocation slip of HfNbTaTiZr BCC-HEA was certainly active on $\{112\}$ planes during tensile deformation at $77 \mathrm{~K}$.

Further, to understand the GB strengthening and strengthductility trade-off at $77 \mathrm{~K}$, additional test was performed at $300 \mathrm{~K}$ for comparison. Fig. 3(c) shows SEM-BSE images of the HfNbTaTiZr deformed at $300 \mathrm{~K}$ after, $e \sim 2.6 \%$. One of the important differences were the coarsely and finely-spaced slip-bands at $77 \mathrm{~K}$ and $300 \mathrm{~K}$, respectively. The coarsely-spaced slip-bands (small number of active slip systems) indicated inhomogeneous slip at $77 \mathrm{~K}$ whereas, finely-spaced slip-bands indicated relatively homogeneous slip and interlaced slip-bands suggested active slip on multiple slip systems at $300 \mathrm{~K}$. In an extension to the Taylors theory, Armstrong et al. [31] suggested that disproportionate of active slip systems can enhance GB strengthening. Later, Rao and Prasad [32] showed GB strengthening of $\mathrm{Mg}$ enhanced at $77 \mathrm{~K}$ due to the activation of prismatic slip, contrary to basal slip at ambient temperatures. In the present study, the preference of dislocation slip on $\{112\}$ planes over the closest packed $\{110\}$ planes might have a similar effect and promote additional GB strengthening in the BCC-HEA at $77 \mathrm{~K}$. Such an effect can in fact compromise the essential requirements for plasticity, especially when the grain size is reduced. This is because, fine grains have less dislocation sources and also accommodates less dislocations in the pile-ups, consequently impose low stress concentration at the GB. Under such circumstances, activation of new dislocation sources across the GB can be difficult which eventually manifests the strength-ductility trade-off similar to the present observations (refer Fig. 1(b)). 


\section{$\underline{T=77 \mathrm{~K}}$}
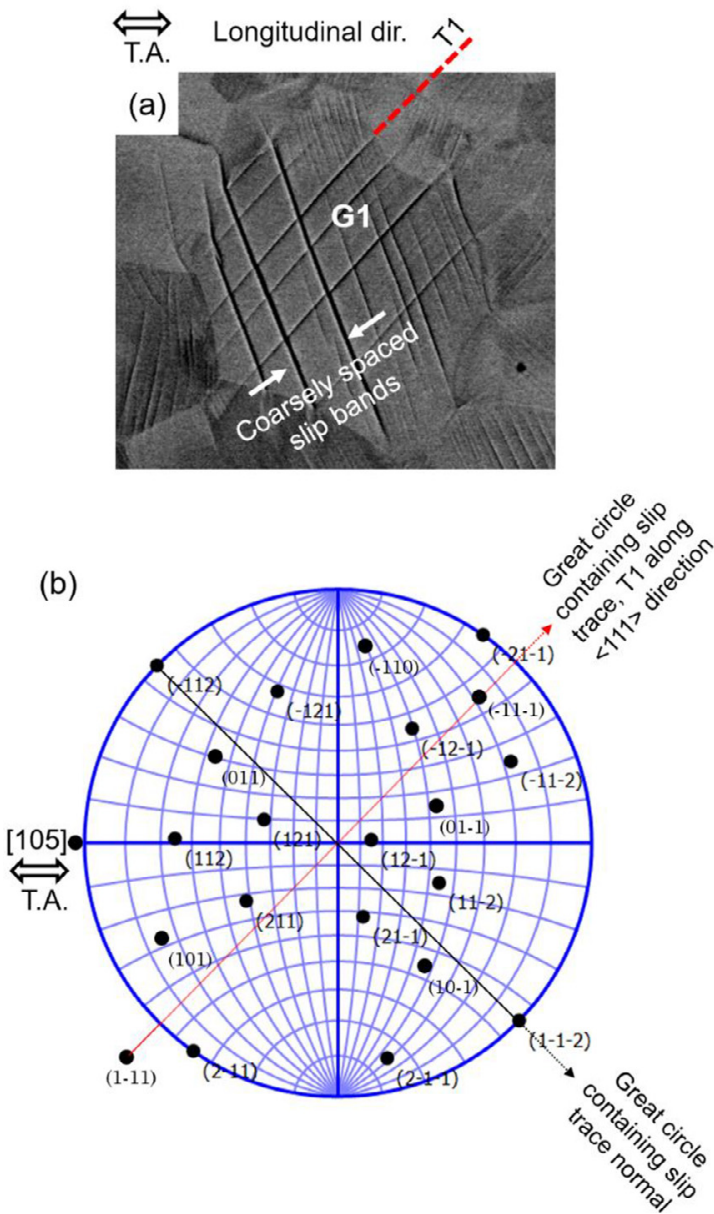

$\underline{T=300 \mathrm{~K}}$

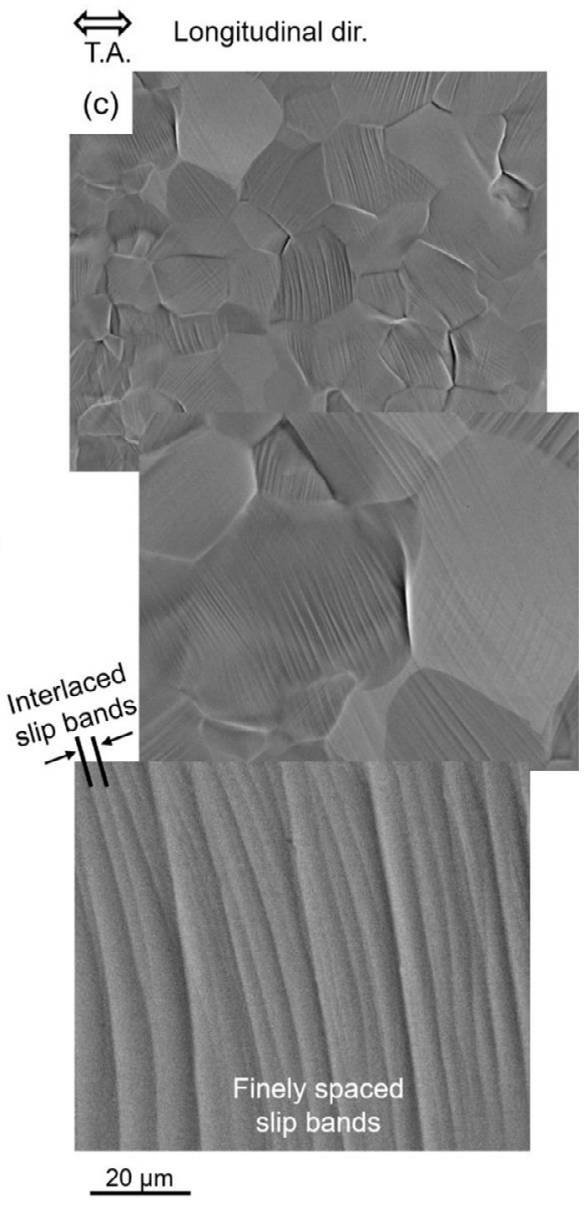

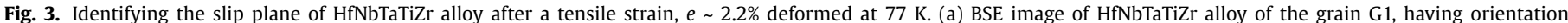

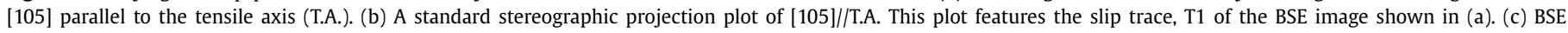
images of HfNbTaTiZr alloy deformed at $300 \mathrm{~K}$ until strain, $e \sim 2.6 \%$.

Later, thermal activation analysis was performed using stress relaxation tests during tensile deformation. The derived apparent activation volume $\left(V^{*}\right)$ was evaluated to compare the characteristics of dislocation slip with those of the available dislocation models. Fig. 4(a) shows stress vs time curve of the HfNbTaTiZr alloy during stress relaxation test at $300 \mathrm{~K}$ and $77 \mathrm{~K}$, for the strain rate $10^{-3} \mathrm{~s}^{-1}$. The details on the evaluation of $V^{*}$ were shown in the supplementary materials. Fig. 4(b) shows the effect of strain on $V^{*}$. The obtained $V^{*}$ values ranged $31-29 \mathrm{~b}^{3}$ at $300 \mathrm{~K}$, and 8.4$7.2 \mathrm{~b}^{3}$ at $77 \mathrm{~K}$. To give perception on the observed $V^{*}$ values, we performed additional tests on pure $\mathrm{Nb}$ (99.9 wt\%). Fig. 4(c) shows stress vs time curve at $77 \mathrm{~K}$. Fig. 4 (d) shows the effect of strain on $V^{*}$. The obtained $V^{*}$ values ranged $7-6.6 \mathrm{~b}^{3}$. As is well known, dislocation motion aided by thermal activation can result in a range of $V^{*}$ values depending on the rate-controlling mechanism. Most notable are, nucleation of kinks $\left(<10 \mathrm{~b}^{3}\right)$, overcoming PeierlsNabarro stress barrier $\left(10 \sim 100 \mathrm{~b}^{3}\right)$, cross-slip and intersection of dislocations (100 1000 $\mathrm{b}^{3}$ ) and cutting through dislocation forest $\left(>1000 \mathrm{~b}^{3}\right)$ [33]. The obtained $V^{*}$ values $\left(31 \sim 29 \mathrm{~b}^{3}\right)$ in HfNbTaTiZr BCC-HEA at $300 \mathrm{~K}$ indicated thermally activated dislocation glide by overcoming the Peierls-Nabarro stress barrier as the ratecontrolling mechanism.

On the other hand, we obtained $V^{*}=8.4-7.2 \mathrm{~b}^{3}$ for the BCC-HEA and almost identical $V^{*}=7.0-6.6 \mathrm{~b}^{3}$ for $\mathrm{Nb}$, at $77 \mathrm{~K}$. Strain independent $V^{*}$ corresponded to low strain-hardening rate.
Similar range of $V^{*}$ values were previously reported in $\mathrm{Nb}$ - [34], Ta- [35] and Fe-based alloys [36]. This implies the same ratecontrolling mechanism for the present HEA and Nb, and other BCC alloys. The closest dislocation model in accordance with the observations was kink nucleation $[6,33,37]$. It is noteworthy that Seeger and Holzwarth [38] systematically studied low-temperature plasticity of $\mathrm{Nb}$ and reported kink/kink-pair nucleation happens on the $\{112\}$ planes. Further, Werner [9] quantitatively analyzed the formation and migration of kinks in Ta, and suggested kink nucleation most likely occurred on the $\{112\}$ planes. In the present study, slip trace analysis of BCC-HEA certainly confirmed slip on the $\{112\}$ planes which merits the plausibility of kink nucleation on the $\{112\}$ planes. However, this aspect requires further investigations.

Later, Rao et al. $[13,14]$ proposed screw dislocation strengthening of BCC-HEAs using Suzuki model which considers migration of kinks is strongly affected by the solute-kink interaction. However, $V^{*}$ values of HfNbTaTiZr BCC-HEA does not suggest the influence of large number(s) of solute(s) of HEA compared to Nb. That is, although the undefined solutes of BCC-HEA may offer shortrange obstacles, the obstacles for kink-glide can easily be overcome by the stress assistance and thermal activation. Another important observation was the very closely related numerical $V^{*}$ values of BCC-HEA and Nb. This implies, the energy barrier for the kink-glide is insensitive to the chemical complexity difference between BCC-HEA and Nb. Furthermore, HEAs are expected to have 
(a)

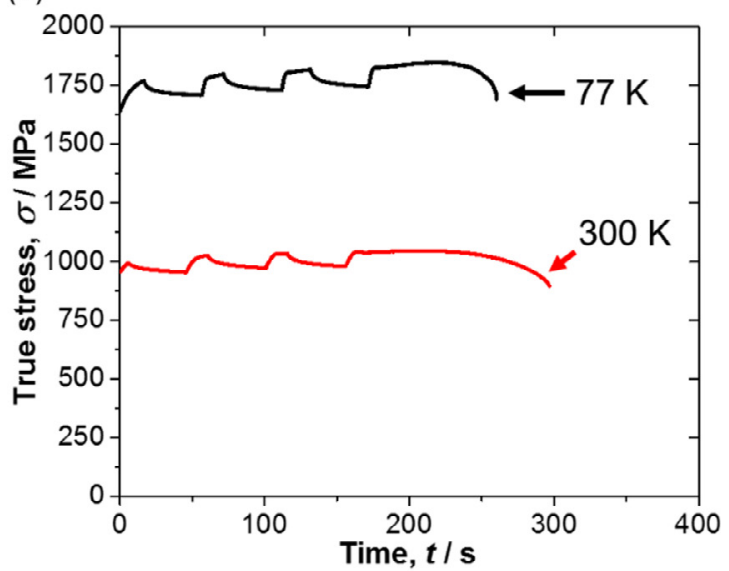

(b)

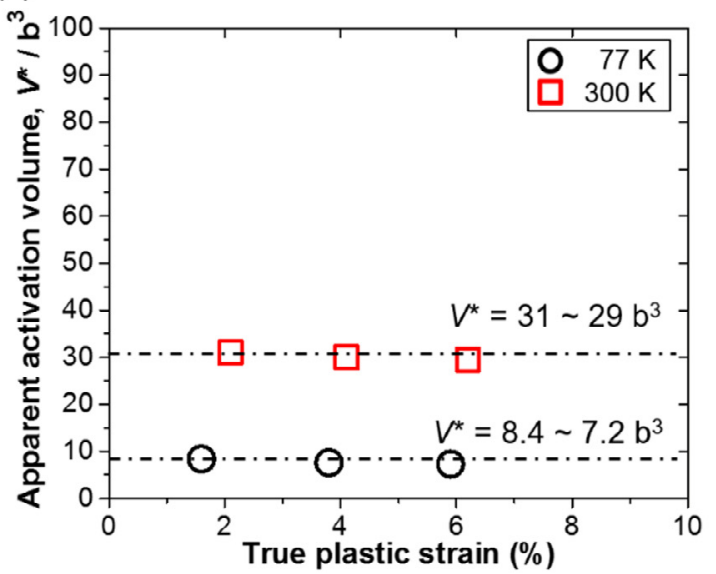

(c)

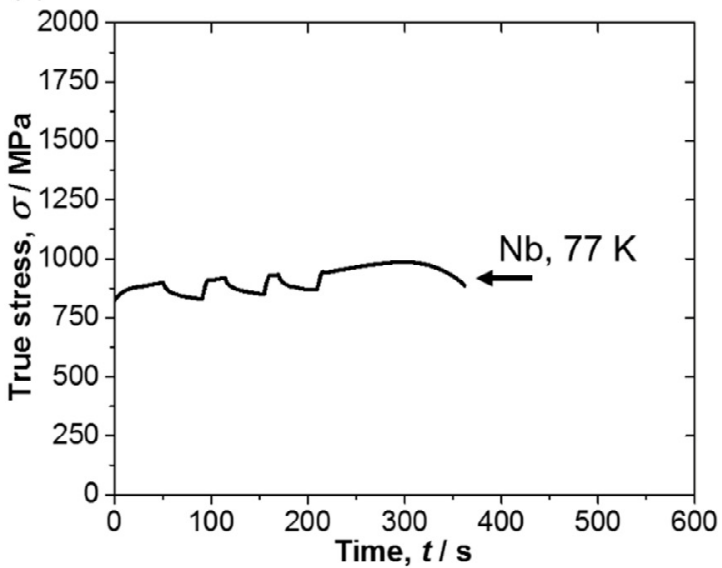

(d)

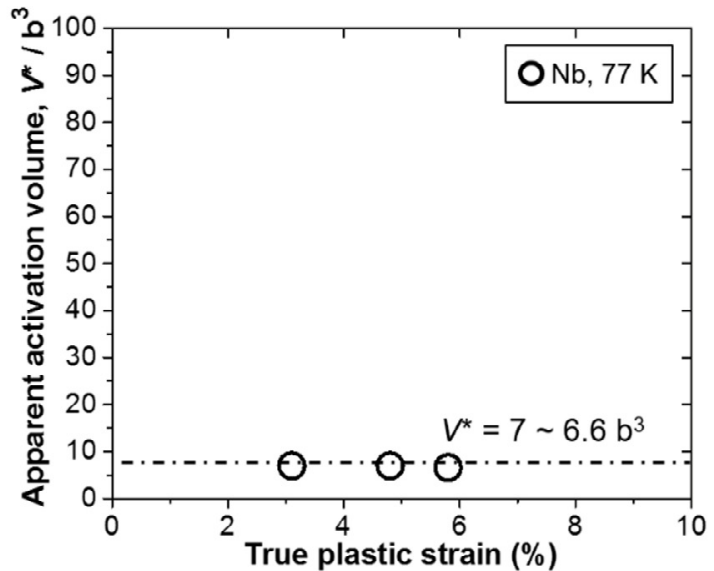

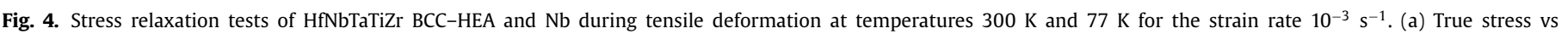

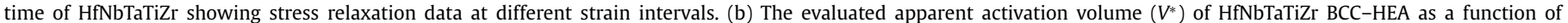

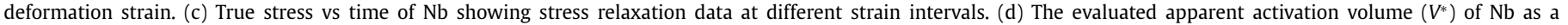
function of deformation strain.

atomic size and shear modulus misfits due to the presence of large concentrations of different elements [39]. Due to this, a dislocation moving through the HEA lattice would experience fluctuated Peierls-Nabarro energy field having hills and valleys of various wavelengths. Nevertheless, present results suggested the thermally activated rate-controlling mechanisms of BCC-HEA and Nb were same. In the sense, the implications of chemical-complexity of HEAs does not affect the fundamental aspects of low-temperature plasticity of the BCC-HEA. In other words, the typical thermal activation of dislocation slip across the Peierls valley suggests the presence of high Peierls-stress (friction stress) in the BCC-HEA is highly unlikely when compared to pure metals or dilute-alloys. Moreover, recent investigations have successfully reproduced the yield strengths of HEAs without using the friction stress term $[15,40-43]$. Thus, the high friction stress derived from the HallPetch plot of Fig. 1(c) is merely the consequence of solid-solution strengthening. Similar analogy was also observed in FCC-HEA. Laplanche et al. [44] reported $V^{*}$ of $\mathrm{CoCrFeMnNi}$ FCC-HEA having $\sim 60 \mathrm{~b}^{3}$ at $77 \mathrm{~K}$ and $\sim 360 \mathrm{~b}^{3}$ at $293 \mathrm{~K}$, which suggested the typical thermally activated dislocation slip. Therefore, it can be concluded that the $a / 2<111>$ screw dislocations of the HfNbTaTiZr BCC-HEA does not necessarily have special type of Peierls potential due to the implications of chemical-complexity of HEAs which alters dislocation mechanism at ambient or sub-zero temperatures.

To summarize, the deformation behavior and thermal analysis of HfNbTaTiZr BCC-HEA were systematically investigated at $77 \mathrm{~K}$.
The tensile strength of HfNbTaTiZr BCC-HEA showed strong temperature dependence, like BCC metals. Low-temperature plasticity was found preferred on $\{112\}$ planes and also indicated inhomogeneous deformation. Moreover, the straight slip-bands suggested stable slip on $\{112\}$ planes. Preference of dislocation slip on $\{112\}$ planes over the $\{110\}$ planes was considered to have promoted additional GB strengthening, which facilitated strength-ductility trade-off. The evaluated apparent $V^{*}$ suggested kink-nucleation and Peierls-type mechanism as the rate-controlling mechanisms at $77 \mathrm{~K}$ and $300 \mathrm{~K}$, respectively, like pure BCC metals. Furthermore, the $V^{*}$ of $\mathrm{Nb}$ and BCC-HEA were almost identical at $77 \mathrm{~K}$. This implied the thermally activated rate-controlling mechanism of HfNbTaTiZr BCC-HEA had the characteristics of pure BCC metals. These results indicated chemical-complexity of HEAs does not necessarily advocate special type of Peierls potential which can alter the dislocation mechanisms in the BCC-HEA.

\section{Declaration of competing interest}

The authors declare that they have no known competing financial interests or personal relationships that could have appeared to influence the work reported in this paper.

\section{Acknowledgements}

The authors gratefully acknowledge the financial support from the Russian Science Foundation Grant no. 19-79-30066. The au- 
thors are grateful to the personnel of the Joint Research Center, "Technology and Materials", Belgorod National Research University, for their assistance. The authors would like to extend acknowledgements to Drs. D. Shaysultanov and N. Yurchenko of Laboratory of Bulk Nanostructured Materials, Belgorod National Research University for their assistance during the experiments.

\section{Supplementary materials}

Supplementary material associated with this article can be found, in the online version, at doi:10.1016/j.scriptamat.2020.07. 028.

\section{References}

[1] C.R. Weinberger, B.L. Boyce, C.C. Battaile, Int. Mater. Rev. 58 (2013) 296-314.

[2] V. Vítek, R.C. Perrin, D.K. Bowen, Philos. Mag. A J. Theor. Exp. Appl. Phys. 21 (1970) 1049-1073

[3] V. Vitek †, Philos. Mag. 84 (2004) 415-428.

[4] D. Brunner, V. Glebovsky, Mater. Lett. 42 (2000) 290-296.

[5] D. Caillard, Acta Mater. 58 (2010) 3504-3515.

[6] D. Caillard, J.L. Martin, Thermally Activated Mechanisms in Crystal Plasticity, first ed., Pergamon, 2003.

[7] A. Seeger, U. Holzwarth†, Philos. Mag 86 (2006) 3861-3892.

[8] H. Conrad, On the Mechanism of Yielding and Flow in Iron, 1961

[9] M. Werner, Phys. Status Solidi 104 (1987) 63-78.

[10] Z.S. Basinski, J.W. Christian, in: K.D. Timmerhaus (Ed.), Springer US, Boston, MA, 1960, pp. 90-92.

[11] K.G. Hoge, A.K. Mukherjee, J. Mater. Sci. 12 (1977) 1666-1672.

[12] J.-.W. Yeh, S.-.K. Chen, S.-.J. Lin, J.-.Y. Gan, T.-.S. Chin, T.-.T. Shun, C.-.H. Tsau, S.-.Y. Chang, Adv. Eng. Mater. 6 (2004) 299-303.

[13] S.I. Rao, B. Akdim, E. Antillon, C. Woodward, T.A. Parthasarathy, O.N. Senkov, Acta Mater. 168 (2019) 222-236.

[14] S.I. Rao, E. Antillon, C. Woodward, B. Akdim, T.A. Parthasarathy, O.N. Senkov, Scr. Mater. 165 (2019) 103-106.

[15] F. Maresca, W.A. Curtin, Acta Mater. 182 (2020) 144-162.

[16] B. Gludovatz, A. Hohenwarter, D. Catoor, E.H. Chang, E.P. George, R.O. Ritchie, Science 80 (345) (2014) 1153 LP -1158.
[17] Z. Wu, H. Bei, G.M. Pharr, E.P. George, Acta Mater. 81 (2014) 428-441.

[18] S. Chen, K.-.K. Tseng, Y. Tong, W. Li, C.-.W. Tsai, J.-.W. Yeh, P.K. Liaw, J. Alloy. Compd. 795 (2019) 19-26.

[19] J. Čížek, P. Haušild, M. Cieslar, O. Melikhova, T. Vlasák, M. Janeček, R. Král P. Harcuba, F. Lukáč, J. Zýka, J. Málek, J. Moon, H.S. Kim, J. Alloy. Compd. 768 (2018) 924-937.

[20] G. Dirras, J. Gubicza, A. Heczel, L. Lilensten, J.-.P. Couzinié, L. Perrière, I. Guillot, A. Hocini, Mater. Charact. 108 (2015) 1-7.

[21] R.R. Eleti, V. Raju, M. Veerasham, S.R. Reddy, P.P. Bhattacharjee, Mater. Charact. 136 (2018) 286-292.

[22] R.R. Eleti, A.H. Chokshi, A. Shibata, N. Tsuji, Acta Mater. 183 (2020).

[23] R.R. Eleti, Deformation Mechanisms and Microstructure Evolution in HfNbTaTiZr High Entropy Alloy During Thermo-Mechanical Processing At Elevated Temperatures Ph.D. Thesis, Kyoto University, Japan, 2019.

[24] R.R. Eleti, T. Bhattacharjee, A. Shibata, N. Tsuji, Acta Mater. 171 (2019) 132-145.

[25] O.N. Senkov, S.L. Semiatin, J. Alloy. Compd. 649 (2015) 1110-1123.

[26] N.D. Stepanov, N.Y. Yurchenko, S.V. Z.herebtsov, M.A. Tikhonovsky, G.A. Salishchev, Mater. Lett. 211 (2018) 87-90.

[27] S. Zherebtsov, N. Yurchenko, D. Shaysultanov, M. Tikhonovsky, G. Salishchev, N. Stepanov, Adv. Eng. Mater. (2020) 2000105 n/a.

[28] C.-.C. Juan, M.-.H. Tsai, C.-.W. Tsai, W.-.L. Hsu, C.-.M. Lin, S.-.K. Chen, S.-.J. Lin, J.-.W. Yeh, Mater. Lett. 184 (2016) 200-203.

[29] S.Y. Chen, L. Wang, W.D. Li, Y. Tong, K.K. Tseng, C.W. Tsai, J.W. Yeh, Y. Ren, W. Guo, J.D. Poplawsky, P.K. Liaw, Mater. Res. Lett. 7 (2019) 475-481.

[30] Z.C. Cordero, B.E. Knight, C.A. Schuh, Int. Mater. Rev. 61 (2016) 495-512.

[31] R. Armstrong, I. Codd, R.M. Douthwaite, N.J. Petch, Philos. Mag. A J. Theor. Exp. Appl. Phys. 7 (1962) 45-58.

[32] G.S. Rao, Y.V.R.K. Prasad, Metall. Trans. A 13 (1982) 2219-2226.

[33] H. Conrad, JOM 16 (1964) 582-588.

[34] C.D. Statham, D.A. Koss, J.W. Christian, Philos. Mag. 26 (1972) 1089-1103.

[35] L.A. Gypen, A. Deruyttere, J. Less Common Met. 86 (1982) 219-240.

[36] Y. Aono, K. Kitajima, E. Kuramoto, Scr. Metall. 15 (1981) 275-279.

[37] G.A. Sargent, Acta Metall. 13 (1965) 663-671.

[38] A. Seeger, U. Holzwarth†, Philos. Mag. 86 (2006) 3861-3892.

[39] M.-.H. Tsai, J.-.W. Yeh, Mater. Res. Lett. 2 (2014) 107-123.

[40] F. Maresca, W.A. Curtin, Acta Mater. 182 (2020) 235-249.

[41] N.L. Okamoto, K. Yuge, K. Tanaka, H. Inui, E.P. George, AIP Adv. 6 (2016) 125008.

[42] G.P.M. Leyson, W.A. Curtin, Model. Simul. Mater. Sci. Eng. 24 (2016) 65005

[43] S.-.P. Wang, J. Xu, Intermetallics 95 (2018) 59-72.

[44] G. Laplanche, J. Bonneville, C. Varvenne, W.A. Curtin, E.P. George, Acta Mater. 143 (2018) 257-264. 